\title{
Computational design of the flow properties of foams
}

\author{
J. Skibinski ${ }^{1}$, T. Wejrzanowski ${ }^{1}$, J. Szumbarski ${ }^{2}$ \& \\ K. J. Kurzydlowski ${ }^{1}$ \\ ${ }^{1}$ Warsaw University of Technology, Faculty of Materials Science and \\ Engineering, Poland \\ ${ }^{2}$ Warsaw University of Technology, Faculty of Power and Aeronautical \\ Engineering, Poland
}

\begin{abstract}
In the present studies the quantitative relationships between the structure and flow properties of porous materials are addressed. In order to investigate such relationships a series of foam structures with different porosity were characterized by computer tomography. Later, 3D image analysis was applied to obtain quantitative parameters of foam structures.

In order to calculate properties of the flow through porous structures, 3D images obtained by computer tomography were numerically processed and transformed into a finite element mesh. Simulation of fluid flow was performed using a Finite Volume Method. Both qualitative characterization and numerical simulation results, when compared, enabled us to establish structure-flow relationships.

The preliminary results show that the pressure drop is strongly related to the porosity of foams. The significant effect on flow properties exhibits also the diversity of pore sizes.

The results will be discussed with respect to foam structure optimization for several applications.

Keywords: ceramic foams, pressure drop, finite volume method.
\end{abstract}

\section{Introduction}

The industrial application of structures with open porosity such as ceramic or metallic foams has been growing over recent decade. Their favorable properties 
such as high specific surface area, high porosity, low density, low thermal conductivity and low specific heat capacity predestine them to serve as compact heat exchangers, reaction catalysts, flow stabilizers or casting filters [1]. These materials can also be found in nature, for example as porous rocks containing gas and oil. Those applications require new materials, which can be designed and investigated numerically before manufacturing. In order to understand quantitative nature of the structure-properties relationships in porous media relevant structural parameters must be defined. Open porosity and relative surface of pores are frequently used for this purpose [2]. These parameters give good general characteristics, however their meaning to the understanding of the material flow properties, such as pressure drop, is rather limited.

Many geometrical models have been developed to describe properties of real foam structures. Most of the models, such as the reproduction of a single type of polyhedron, or models based on Kelvin polyhedron (gives minimum specific surface) [3], exhibit anisotropy, which usually does not exist in real foams. Most of cellular structures are fabricated by techniques based on growth process. Poisson-Voronoi tessellation (PVT) is frequently used as an algorithm to obtain representative cellular structures of this type. However, geometrical parameters of real foams, such as average faces per cell, differ from those obtained by PVT. A representation of real foams structure can be obtained by application of Laguerre-Voronoi algorithm, where tessellations are performed on the set of spheres with pre-determined size distribution $[4,5]$.

This paper shows the preliminary numerical results of the modelling of fluid flow properties through real structures and the model structures obtained by Laguerre-Voronoi tessellation.

\section{Geometries}

\subsection{Real structures}

The commercially available alumina foams were used as a reference foam structures (further called real structures). The real structures were originally described by pores per inch number (ppi). Further $3 \mathrm{~d}$ image analysis enabled for extended characterization, where other structural parameters such as porosity, specific surface, mean pore diameter, mean strut diameter were calculated.

Digitalized geometries of foams were obtained using Computer Tomography (CT). The vector-like structures were created from set of bitmaps. The surface of the foam is divided into a logical series of triangles. Each triangle is uniquely defined by its normal and three vertices. To create a valid volume, the surface geometry was converted to file of points cloud to simplify model in problematic areas. As a result, a valid 3D multi-surface mesh was created. The distribution of the strut diameter as well as specific surface area of each sample were calculated during post-processing. 
Real ceramic foams have varying percentage of pores that are blocked. This is the effect of fabrication process, and the percentage of clogged pores increases with number of pores per inch. The resistance to the flow was expected to be higher in the structures with higher number of blocked pores.

In figure 1 a real geometry and its virtual representation is shown. Table 1 lists structural parameters of foams obtained after processing.

Table 1: $\quad$ Parameters of real ceramic foams.

\begin{tabular}{|c|r|c|c|}
\hline Structure & $\begin{array}{c}\text { Porosity } \\
{[-]}\end{array}$ & $\begin{array}{c}\text { Specific surface area } \\
{[1 / \mathrm{mm}]}\end{array}$ & $\begin{array}{c}\text { Mean strut } \\
\text { diameter } \\
{[\mathrm{mm}]}\end{array}$ \\
\hline $10 \mathrm{ppi}$ & 0.740 & 0.77906 & 1.35222 \\
\hline $30 \mathrm{ppi}$ & 0.702 & 1.22055 & 0.89627 \\
\hline $50 \mathrm{ppi}$ & 0.754 & 1.84731 & 0.57974 \\
\hline
\end{tabular}
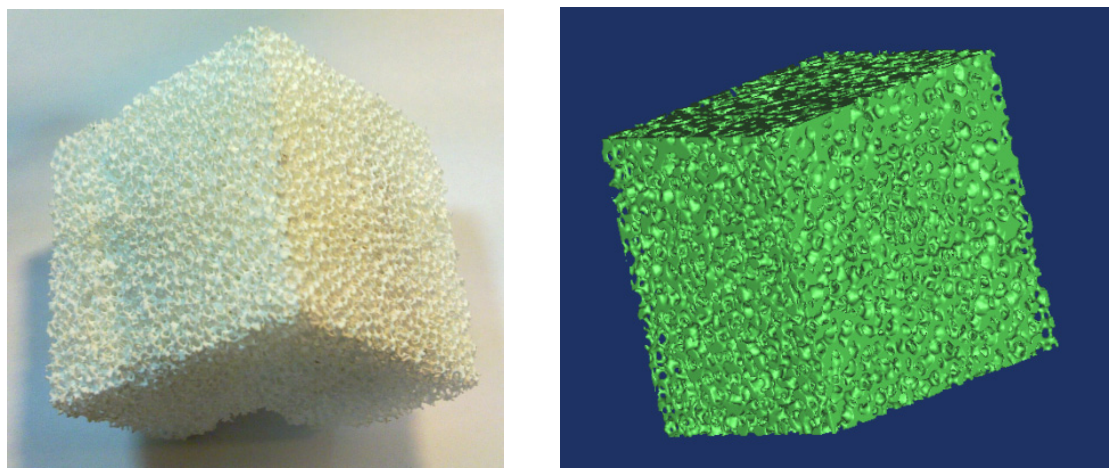

Figure 1: $\quad$ Ceramic foam and its computer model.

\subsection{Foam numerical models}

Foam models were created using Laguerre-Voronoi tessellation (LVT). LVT was applied to spheres with specified size distribution, which were packed in the cubic volume. The foam struts were created by cylinders with defined constant diameter generated along cell edges (see Figure $2 \mathrm{a}$ ). 


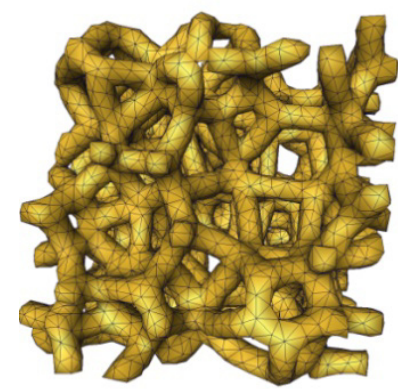

a) Model structure

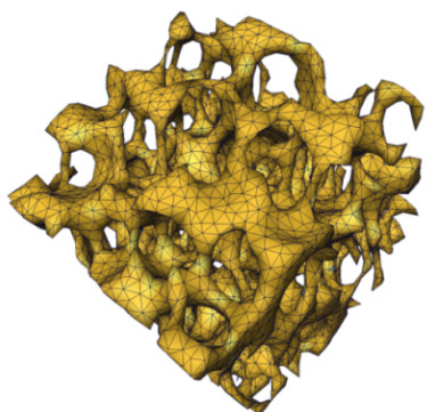

b) $\mathrm{CT}$ reconstructed real structure

Figure 2: $\quad$ Computer models of designed structure (a) and real structure (b).

Parameters of structures designed within these studies are shown in Table 2.

Table 2: $\quad$ Parameters of foam models.

\begin{tabular}{|c|c|c|c|}
\hline Structure & $\begin{array}{c}\text { Porosity } \\
{[-]}\end{array}$ & $\begin{array}{c}\text { Specific surface area } \\
{[1 / \mathrm{mm}]}\end{array}$ & $\begin{array}{c}\text { Mean strut diameter } \\
{[\mathrm{mm}]}\end{array}$ \\
\hline CV 01 & 0.6964 & 0.76741 & 1.544 \\
\hline CV 03 & 0.7480 & 0.75987 & 1.278 \\
\hline Bimodal & 0.8157 & 0.47446 & 1.459 \\
\hline
\end{tabular}

The effect of pore clogging does not appear in the model structures. Blocked pores don't change porosity significantly, but the pressure drop is expected to be smaller regardless structural parameters.

\section{Numerical method}

Fluid flow calculations were performed using Finite Volume Method. This method represents partial differential equations in the form of algebraic equations. Values are calculated at discrete place on a meshed geometry. The key to the method is that the integral form of the conservation law (1) can be rewritten using the Gauss Divergence Theorem.

$$
\frac{\partial}{\partial t} \int_{\Omega} U d \Omega+\int_{\Omega} \vec{\nabla} \cdot \vec{F} d \Omega=0
$$

Volume integrals of divergence terms in a partial differential equation are converted to surface integrals of fluxes all around the control volume (2).

$$
\frac{\partial}{\partial t} \int_{\Omega} U d \Omega+\oint_{\partial \Omega} \vec{F} \cdot \vec{n} d \Gamma=0
$$

The resulting equations cannot be solved analytically and a numerical solution requires discretization into volumes. These terms are then evaluated as fluxes at the surfaces of each finite volume. The flux entering a given volume is 
identical to that leaving the adjacent volume. This makes the FVM stable and flexible, yet relatively easy to implement. The main advantage of this method is it's applicability on unstructured meshes and the intrinsic local conservation properties of the resulting schemes, which suits very well the irregular geometries of porous structures.

The flows across real and designed structures were simulated. The computational domain was a channel with length of $20 \mathrm{~mm}$ and $15 \times 15 \mathrm{~mm}$ square cross-section. Structural parameters of specimens are shown in Table 2. The size of specimens was $15 \mathrm{~mm} \times 15 \mathrm{~mm} \times 15 \mathrm{~mm}$ block. The inlet velocities for FVM simulations range from 0.0002 to $0.02 \mathrm{~m} / \mathrm{s}$, which correspond to Reynolds number from 2 to 400 .

\section{Results}

The results of the simulations of flows across real foams are compared with the designed structures. The hydraulic resistance of all structures is shown in Figure 3.

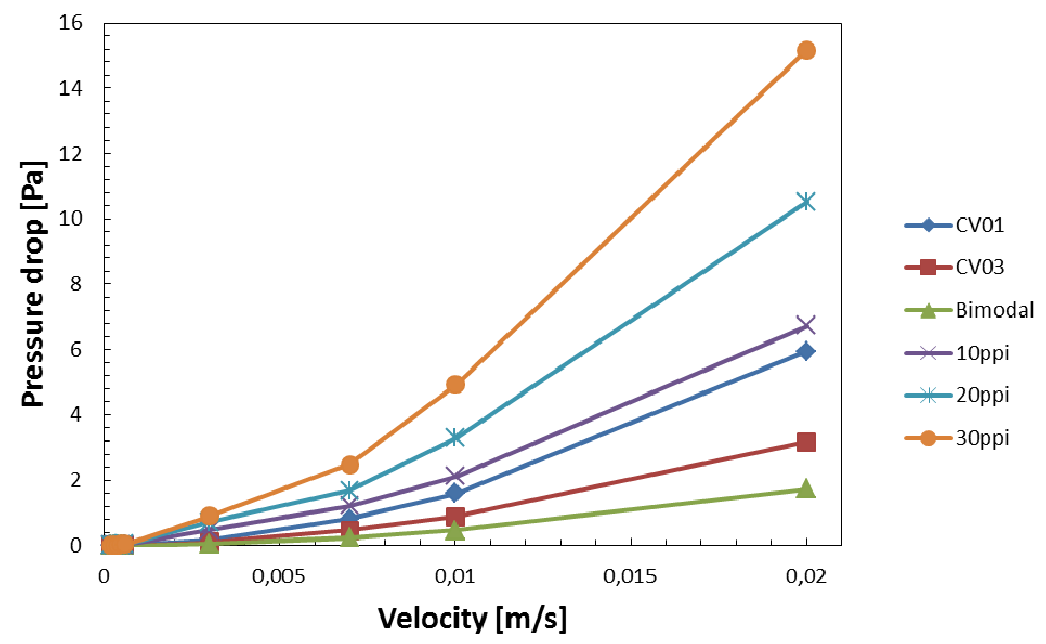

Figure 3: Comparison of simulated and experimental pressure drop in flow across different ceramic foams.

The structures that exhibit the closest similarity regarding structural parameters are 10ppi and CV03. These structures have been chosen for comparison of hydraulic properties of real and designed materials (Figure 4). 


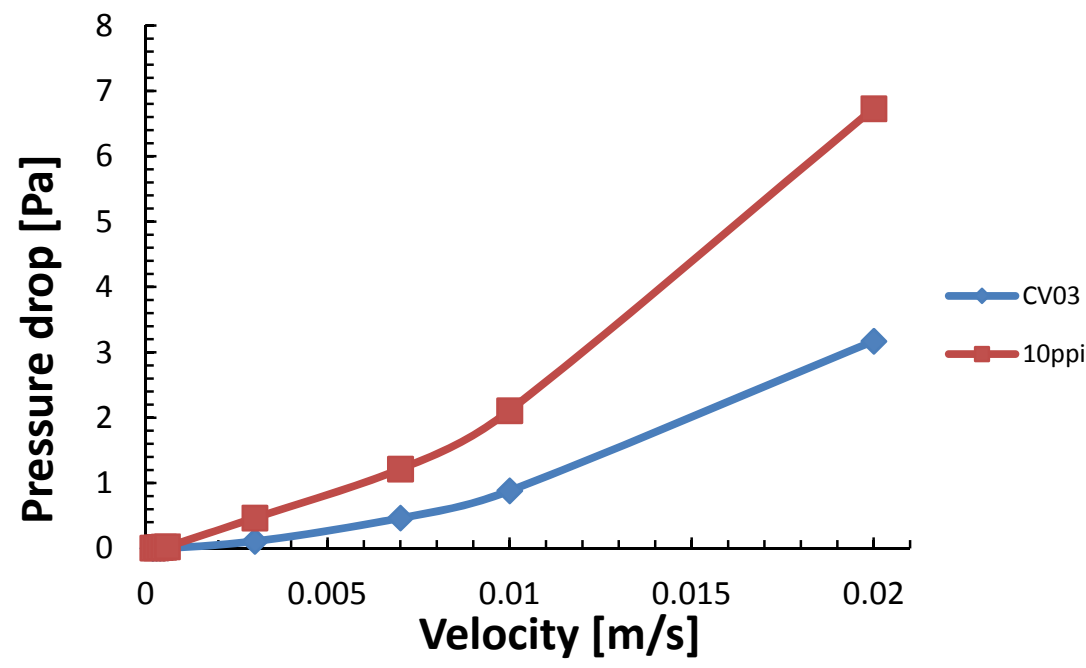

Figure 4: Comparison of pressure drop in flow across designed and real structures with similar structural parameters.

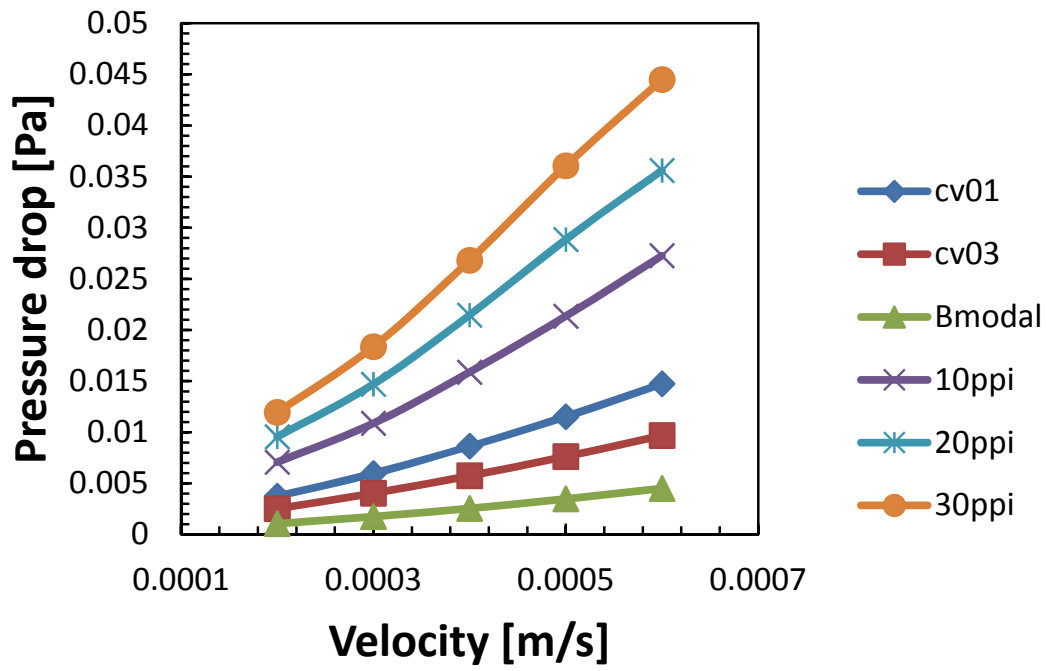

Figure 5: $\quad$ Pressure drop results for Darcy's flow regime.

Linear Darcy's model is most commonly used for describing flows through porous media. Darcy's law may be written as: 


$$
-\frac{d p}{d L}=\frac{\mu}{k} \cdot \vec{v}
$$

where $\mathrm{dL}$ is a segment [m] along which a pressure drop dp [Pa] occurs, $\mathrm{k}$ permeability $\left[\mathrm{m}^{2}\right], \mu$ dynamic viscosity $[\mathrm{kg} / \mathrm{ms}]$ and $\mathrm{v}$ is the filtration velocity $[\mathrm{m} / \mathrm{s}]$. Darcy's law correctly describes the flow in porous media only for low velocity flows. As a basic criterion used to describe correct Darcy's regime is Reynolds number. The range of Reynolds number for Darcy's flow differs in dependence of sources [12-15], but $\mathrm{Re}=10$ is generally assumed to be a critical maximum value. Set of calculation results based on this criterion have been chosen to determine effective permeability of materials. Reynolds number valid for Darcy's law is equivalent to velocities of $0.0002 \mathrm{~m} / \mathrm{s}$ to $0,0006 \mathrm{~m} / \mathrm{s}$ for porous foams sizes used. Figure 5 shows the results of pressure drop for selected range of velocities.

For flows with Reynolds number exceeding the Darcy's law criterion, a discrepancy between experimental data and results obtained based on Darcy's law appears [16]. Forchheimer linked the latter to kinetic effect and suggested to modify (3) by an additive term $\rho \bar{u}^{2}$, representing kinetic energy [17]:

$$
-\frac{d p}{d L}=\frac{\mu}{\kappa} \cdot \vec{v}+\beta \rho \vec{v}
$$

where $\beta[1 / \mathrm{m}]$ is Forchheimer's coefficient (also known as non-Darcy coefficient). The results of pressure drop in investigated structures for non-Darcy flow are shown in Figure 6.

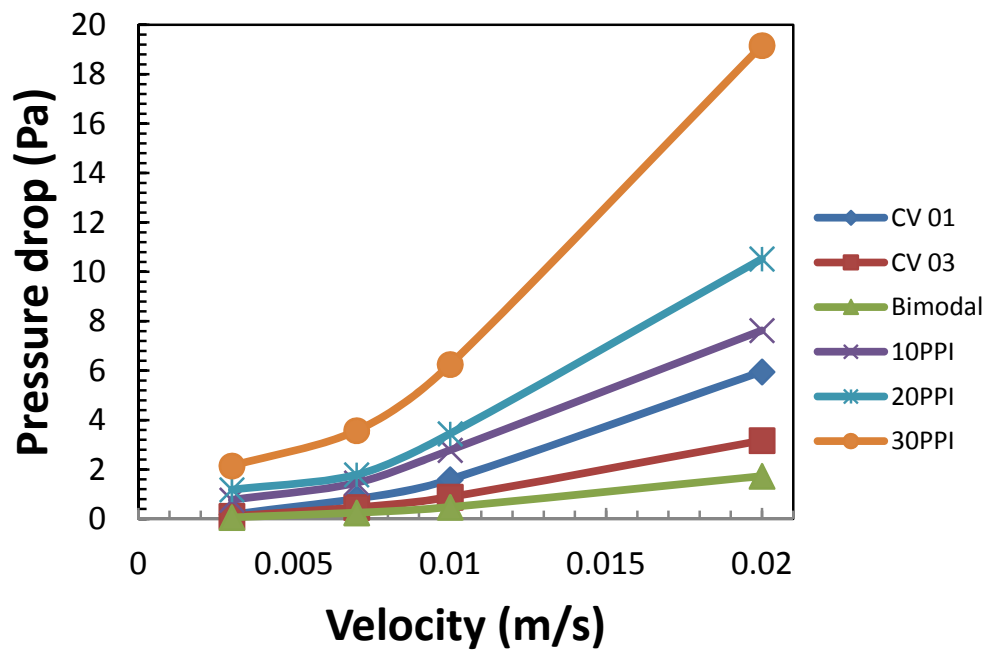

Figure 6: $\quad$ Pressure drop in porous structures for non-Darcy flow.

There is a good agreement between obtained results and the basic models describing flows through porous media. When the Reynolds number exceeds the 
maximum value for Darcy's flow, the pressure vs. velocity curve changes from linear to polynomial for every investigated structure, which accords with Forchheimer's equation (4).

The effective permeability $k_{\text {eff }}$ of materials have been determined from Darcy's law. The results are shown in Figure 7.

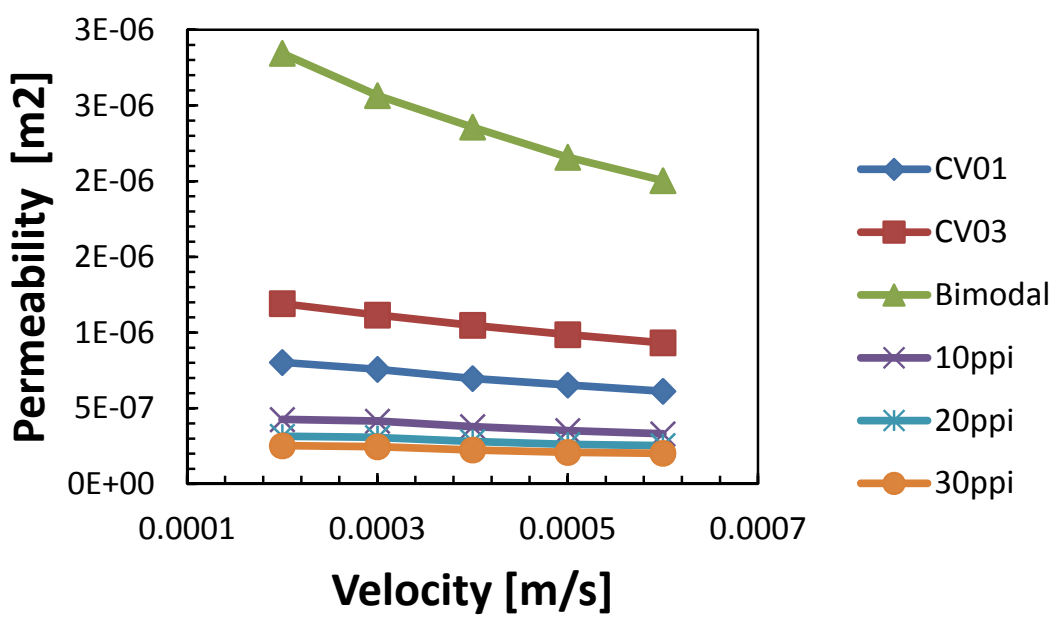

Figure 7: Effective permeability coefficient in function of velocity.

In general, $\mathrm{k}_{\text {eff }}$ takes into account not only permeability coefficient characterizing the medium, but also inertial effects. As the contribution of inertial effects grows with increasing velocity of flow, the effective permeability decreases.

\section{Conclusions}

Simulations of flows across porous structures were performed to determine hydraulic properties. Pressure drop difference between real and designed structures can be observed. This effect may be related with clogged pores, a result of the production process. The equivalent model structures considered in the simulations should be modified to implement blocked pores in the design process, which should result in more accurate model of real structure.

\section{Acknowledgement}

The work has been financially supported by the Polish Ministry of Science and Higher Education, research grant no. N N507 273636. 


\section{References}

[1] A. Oscilowski, W. Plis, Ceramic foam filters - using purpose, practical experience in Ferro-Term, Archives of Foundry, Vol. 1, Book 1, PAN Katowice, 2001.

[2] Edouard D., Lacroix M., Huu C.P., Luck F., Pressure drop modeling on SOLID foam: State-of-the art correlation. Chem. Eng. Journal 144, pp. 299311, 2008.

[3] Weaire, D.; Phelan, R. (1994), “A counter-example to Kelvin's conjecture on minimal surfaces", Phil. Mag. Lett. 69: 107-110.

[4] Z. Fan, Y. Wu, X. Zhao, Y. Lu, Simulation of polycrystalline structure with Voronoi diagram in Laguerre geometry based on random closed packing of spheres, Computational Materials Science 29 (2004) 301-308.

[5] C. Redenbach, Microstructure Models for Cellular Materials, Computational Materials Science 44, 4 (2009) 1397-1407.

[6] Timothy Bart, Mario Ohlberger, Finite Volume Methods: Foundation and Analysis, Encyclopedia of Computational Mechanics, Chapter 9, 2004.

[7] M.R. Nangrejo, X. Bao, M.J. Edirisinghe, The structure of ceramic foams produced using polymeric precursors, Journal of Materials Science Letters 19 (2000) 787-789.

[8] J.G. Fourie, J.P. DuPlessis, Pressure drop modelling in cellular metallic foams, Chem. Eng. Sci. 57 pp. 2781-2789, 2002. [7] F.C. Buciuman, B. Kraushaar-Czarnetzki, Ceramic foam monoliths as catalyst carriers. 1. Adjustment and description of the morphology, Ind. Eng. Chem. Res. 42 pp. 1863-1869, 2003.

[9] V.N. Antsiferov, S.E. Porozova, Foam ceramic filters for molten metals: reality and prospects, Powder Metallurgy and Metal Ceramics, Vol. 42, Nos. 9-10, 2003.

[10] Luis Seminario, Roberto Rozas, Rodrigo Borquez, Pedro G. Toledo, Pore blocking and permeability reduction in cross-flow microfiltration, Journal of Membrane Science 209 (2002) 121-142.

[11] A. Santos, P. Bedrikovetsky, S. Fontoura, Analytical micro model for size exclusion: Pore blocking and permeability reduction,. Journal of Membrane Science 308 (2008) 115-127.

[12] Bear J., Dynamics of Fluids in Porous Media, Elsevier, 1972.

[13] Hansen T. E., Flow in micro porous silicon carbide. Master Thesis, Department of Micro and Nanotechnology, Technical University of Denmark, March 2nd, 2007.

[14] Hassanizadeh S.M., Gray W.G., High Velocity Flow in Porous Media, Transport in Porous Media (1987) 2: 521-531.

[15] Sawicki J., Szpakowski W., Weinerowska K., Woloszyn E., Zima P., Laboratory of Fluid Mechanics And Hydraulics, Gdansk University of Technology, 2004.

[16] Andrade J. S., Costa U. M. S., Almeida M. P., Makse H. A., Stanley H. E., Inertial Effects on Fluid Flow through Disordered Porous Media, Physical Review Letters, Vol. 82, No. 26, 28 June 1999. 
[17] Ewing R., Lazarov R., Lyons S.L., Papavassiliou D.V., Pasciak J., Qin G.X., Numerical Well Model For Non-Darcy Flow, Computational Geosciences. 3(3-4). 185-204. 1999. 